Mini Review

\title{
Classification of Renal Proliferative Lesions in Rats and/or Mice and Their Diagnostic Problems: Report from the Working Group of the Japanese Society of Toxicologic Pathology
}

\author{
Kunitoshi Mitsumori ${ }^{1}$, Midori Yoshida ${ }^{2}$, Hijiri Iwata ${ }^{3}$, Osamu Katsuda ${ }^{4}$, \\ Mami Kouchi ${ }^{5}$, and Hiroyuki Tsuda ${ }^{6}$ \\ ${ }^{1}$ Laboratory of Veterinary Pathology, Tokyo University of Agriculture and Technology, 3-5-8 Saiwai-cho, Fuchu, Tokyo \\ 183-8509, Japan \\ ${ }^{2}$ Department of Pathology, Sasaki Institute, 2-2 Kanda-Surugadai, Chiyoda-ku, Tokyo 101-0062, Japan \\ ${ }^{3}$ Department of Pathology, Biosafety Research Center, Foods, Drugs and Pesticides, 582-2 Shioshinden, Fukude-cho, \\ Iwata-gun, Shizuoka 437-1213, Japan \\ ${ }^{4}$ Kashima Laboratory, Mitsubishi Chemical Safety Institute Ltd., 14 Sunayama, Hasaki-machi, Kashima-gun, Ibaraki \\ 314-0255, Japan \\ ${ }^{5}$ Department of Pathology, Dainippon Pharmaceutical Co., Ltd., Enoki 33-94, Suita, Osaka 564-0053, Japan \\ ${ }^{6}$ Experimental Pathology and Chemotherapy Division, National Cancer Center Research Institute, 5-1-1 Tsukiji, \\ Chuo-ku, Tokyo 104-0045, Japan
}

\begin{abstract}
The Working Group for the Proliferative Lesions of the Kidney in Rats and Mice was established by the Japanese Society of Toxicologic Pathology (JSTP) to discuss diagnostic problems on renal proliferative lesions in rodents. Members of the Working Group brought many histological specimen and literatures, and compared them with the nomenclatures of renal proliferative lesions in rats recommended by the groups of STP, WHO/IARC/RITA and NACAD proposed in 2000. The Working Group recognized that both of spontaneous and chemical-induced lesions could be categorized by the use of the same diagnostic criteria and classification based on histological and cytological characteristics. The results of histochemical and immunohistochemical examinations indicate that the origins of epithelial proliferative lesions are proximal tubules for basophilic cell type, either proximal or distal renal parts for their clear cell counterpart, and collecting ducts for oncocytic one, whereas the origin of clear cell type is still controversial. The Working Group proposed the modified classification of renal proliferative lesions that were slightly different from the recommendation of international harmonization of rat nomenclature of the kidney proposed by the groups of STP WHO/IARC/RITA and NACAD. (J Toxicol Pathol 2002; 15: 175-190)
\end{abstract}

Key words: classification, kidney, proliferative lesions, rodent

\section{Introduction}

Renal tumors in rats and mice can be classified into three broad categories depending on whether their cellular compositions such as the epithelium, connective tissues, or primordial embryonic tissues. Renal tumors in rodents, particularly rats, have been induced by the exposure to a variety of agents, namely chemical carcinogens, irradiation or oncogenic virus ${ }^{1,2}$, whereas their spontaneous occurrence is rare. On the other hand, the possibility that chronic

Received: 15 July 2002, Accepted: 15 August 2002

Mailing address: Midori Yoshida, Department of Pathology, Sasaki

Institute, 2-2 Kanda-Surugadai, Chiyoda-ku, Tokyo 101-0062, Japan

TEL: 81-3-3294-3286 FAX: 81-3-3294-3290

E-mail: pathol@sasaki.or.jp treatment with some nephrotoxic chemicals lead to renal neoplasm development could not ruled out ${ }^{3-5}$. Therefore, the underlying renal tumor development is important not only for the research on renal carcinogenesis but also the aspects of toxicologic pathology. So far diagnostic criteria for renal proliferative lesions in rodents have been published in several textbooks on tumor pathology in rats and mice $e^{1,6,7}$, but their nomenclatures are not always the same. In 2000, the working group of Society of Toxicologic Pathology (STP), World Health Organization (WHO)/International Agency for Research on Cancer (IARC)/Registry of Industrial Toxicology Animal Data (RITA) and North American Control Animal Database (NACAD) (http:// www.toxpath.org/) has proposed the recommendation of international harmonization of rat nomenclature of the urinary system, kidney. 
In 2001, the Japanese Society of Toxicologic Pathology (JSTP) decided to establish "the Working Group for the Proliferative Lesions of the Kidney in Rats and Mice" as the special issue on the Continuing Education Program for toxicologic pathologists in Japan, since the Educational Committee of JSTP felt that there are still several points that need to be clarified in the internationally harmonized nomenclature recommended. The Working Group consisting of two pathologists from the academia, two from the non-profit organizations and two from the private contract or pharmaceutical laboratory brought histopathological specimen as well as literatures on renal proliferative lesions in rats and mice, and met 5 times by November 2001 to discuss diagnostic problems that are different from the nomenclatures of renal proliferative lesions in rats recommended by the groups of STP, WHO/ IARC/RITA and NACAD. The outcome of the discussion of the Working Group was presented in the second meeting for the Continuing Education Seminar for Toxicologic Pathologists on November 29, 2001 in Nagoya.

In this report, we introduced pathobiological features of renal tumors focusing on epithelial and primordial embryonic ones, because they are rather common in rodents. Then, we proposed our modified classification and nomenclature of renal proliferative lesions based on the histological and cytological features on their lesions collected by the Working Group of the JSTP. Additionally, some comments were made on crucial problems for the diagnosis of renal lesions, such as distinction between regenerative renal tubules and atypical hyperplasia in chronic progressive nephropathy and their interpretation. Finally, we compared our classification with the final recommendation of international harmonization of rat nomenclature of the kidney proposed by the groups of STP, WHO/IARC/RITA and NACAD in 2000.

\section{Results and Discussion}

\section{Morphological features of renal epithelial proliferative lesions}

Renal proliferative lesions derived from tubular epithelial cells are classified into two types by histological and cytological aspects, the latter being based on intensity in hematoxylin-eosin staining.

\section{1) Histological classification}

Histologically, epithelial preneoplastic and neoplastic lesions are classified into two types, epithelial and tubular types (Fig. 1).

(1) Epithelial type (Plates 1 and 2): This type was the most common change in renal epithelial lesions observed in both chemical-induced and spontaneous cases. It was characterized by intra-tubular proliferation of epithelial cells with papillary, solid, cystic or mixed growth (Fig. 2).

(2) Tubular type (Plate 1D): The lesion of this type was found as a focal proliferation of epithelial or oval like cells

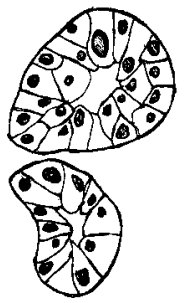

Epithelial type

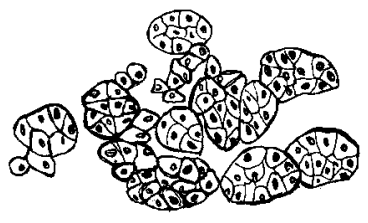

Tubular type
Fig. 1. Histological classification.
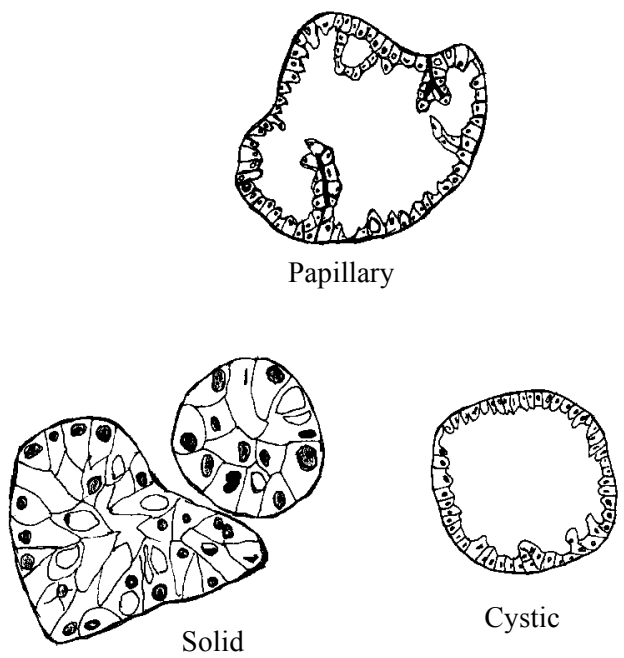

Fig. 2. Sub-classification of epithelial type.

consisting of small tubules or tubule-like structures in the interstitial tissue without obvious compression of surrounding tissue, but the lesions never proliferated within the tubules (Fig. 1). The lesion was observed in the severe damaged kidney where normal architecture was almost destroyed. Some nephrotoxic chemicals are known to induce the lesion although spontaneous occurrence of the lesion is rare except for severely affected kidney with aging such as advanced chronic progressive nephropathy ${ }^{3,5}$. Histogenesis of this type cell remains to be unclear, however this lesion has been considered to have the multi-potent capability to differentiate to tubular or proximal tubular epithelial cell ${ }^{3}$. Interestingly, it was also observed in the chronic dialysis kidney in humans, indicating that histogenesis of this lesion might be common in humans and rats, although further investigations are required for underlying histogenesis of this lesion.

\section{2) Cytological classification}

Based on cytological aspect using hematoxylin-eosin staining, epithelial type described above could be subclassified into three patterns, basophilic (sometimes eosinophilic) cell, clear cell or oncocytic types.

(1) Basophilic/eosinophilic cell type (Plate 2A and 2B): This cell type was the most popular pattern in epithelial 

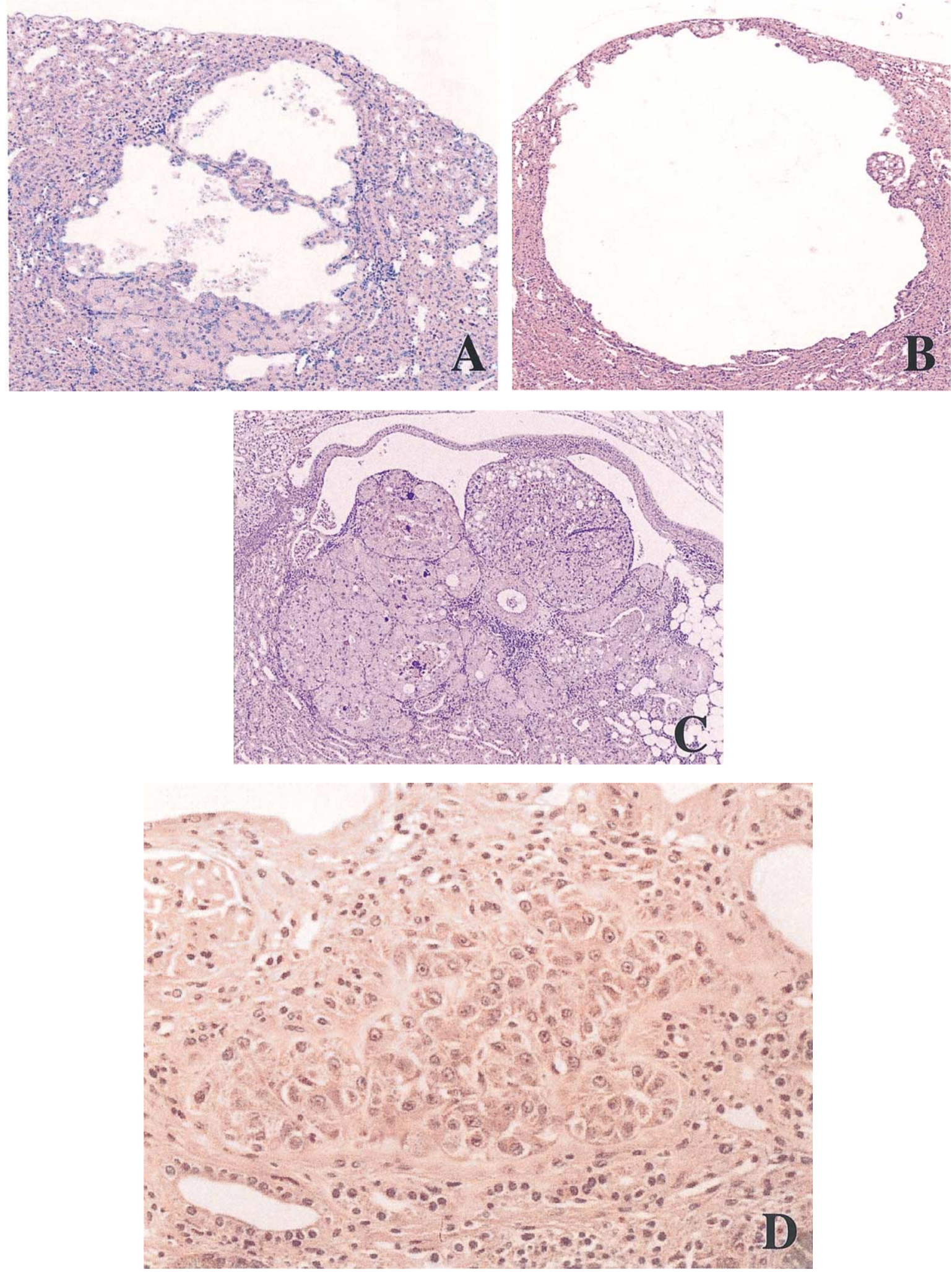

Plate 1. Histological classification. A-C; epithelial types. A; papillary with solid type, B; cystic type and, C; solid type. D; tubular type. Note focal proliferation of epithelial or oval like cells consisting of clusters of small tubules or tubule-like structures in the interstitium of the damaged kidney where normal architecture was almost destroyed. A-D, induced cases. HE stain. 

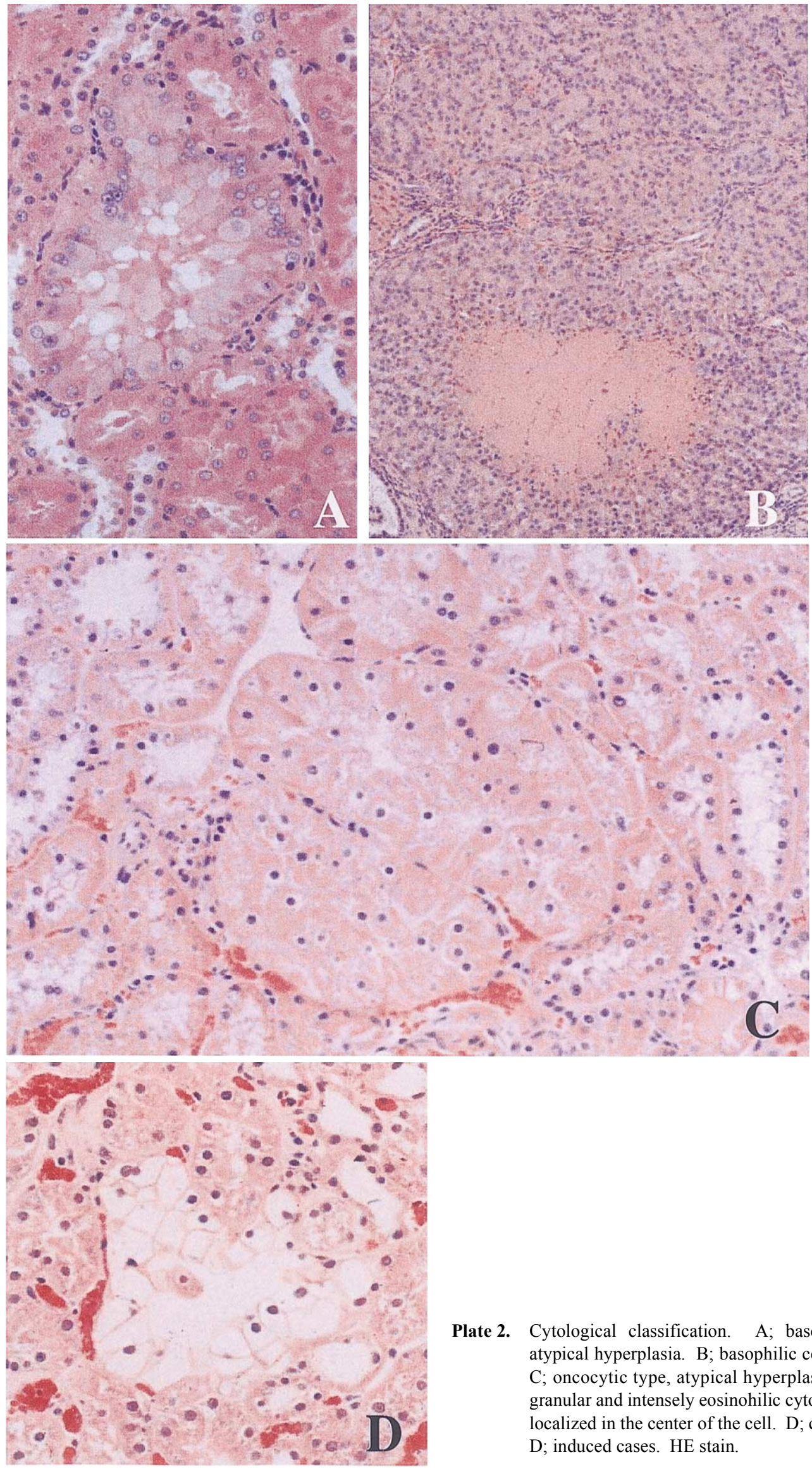

Plate 2. Cytological classification. A; basophilic cell type, atypical hyperplasia. B; basophilic cell type, adenoma. C; oncocytic type, atypical hyperplasia. Note a finely granular and intensely eosinohilic cytoplasm with nuclei localized in the center of the cell. D; clear cell type. A$D$; induced cases. HE stain. 
proliferative lesions. This type was found as a focus of cells with increased basophilia in their cytoplasm compared to the adjacent non-neoplasitc renal tissues. Intensity of hematoxylin-eosin staining was often influenced by many factors such as fixation time or fixation method, and therefore this cell type sometimes looked like having eosinophilic property in the cytoplasm rather than basophilic one.

(2) Clear cell type (Plate 2D): This type was well distinguished from the surrounding tissue by cells with clear cytoplasm.

(3) Oncocytic type (Plate 2C): Oncocytic type was a unique cell type with a finely granular and intensely eosinophilic cytoplasm. The nucleus of oncocyte was large, round or oval, and localized in the center of the cell. Typically, the chromatin displayed some condensed portions closely associated with the nuclear membrane and the nucleolus but was sparsely distributed throughout the remaining karyoplasm. The nuclei sometimes appeared to be pyknotic. Spontaneous occurrence of this type was rare, but it is known that this type lesion is induced by some chemical carcinogens ${ }^{5}$.

\section{Histochemical and immunohistochemical characteristics in renal epithelial proliferative lesions (Plate 3)}

Generally, the overall histochemical and immunohistochemical characteristics in preneoplastic and neoplastic lesions correspond to those of normal cell counterparts in many aspects. Similarly, it has been reported that alterations of certain enzyme expression in the kidney are evident with renal tumor development ${ }^{8}$. Therefore, the detection of enzymes expressed specially in the renal tubular epithelium would be a useful tool for determination of the histogenesis of renal proliferative lesions. The distribution of the enzymes located in the renal tubular cell is schematically demonstrated in Fig. 3. The activities of the enzymes concerning glycogen metabolic pathway including succinate dehydrogenase (SDH) and glucose-6-phosphase dehydrogenase (G6PD), and also $\gamma$-glutamyl transpeptidase (GGT) which is distributed in brush border of proximal epithelial cells can be measured as parameters to demonstrate phenotypic cellular changes. Periodic acid Shiff (PAS) reaction is also a useful histochemical tool to detect the presence of brush border in the proximal epithelium. In addition, Tsuda and his co-workers examined following enzymes as additional parameters: Carbonic anhydrase type II (CA), a marker of lower nephron segments, especially collecting ducts, and cytochrome oxidase (CytOx), a final enzyme of the mitochondrial respiratory chain ${ }^{9}$. These enzymes were clearly positive in the distal and collecting tubules but not in the proximal tubules.

The histochemical and immunohistochemical features of normal renal tubules and renal epithelial tumors including preneoplastic lesions induced by $\mathrm{N}$-nitrosomorpholine (NNM) or N-ethyl-N-hydroxyethylnitrosoamine (EHEN) in rat are shown in Table 1, which is referred to the data reported by Tsuda et al. ${ }^{8,9}$.

Basophilic/eosinophilic epithelial proliferating lesions proved to be strongly positive for G6PD, while demonstrating a reduction or loss of GGT and SDH compared with controls and the surrounding proximal or distal tubules. The result suggests that an increased pentose phosphate pathway and glycolysis characterize basophilic/
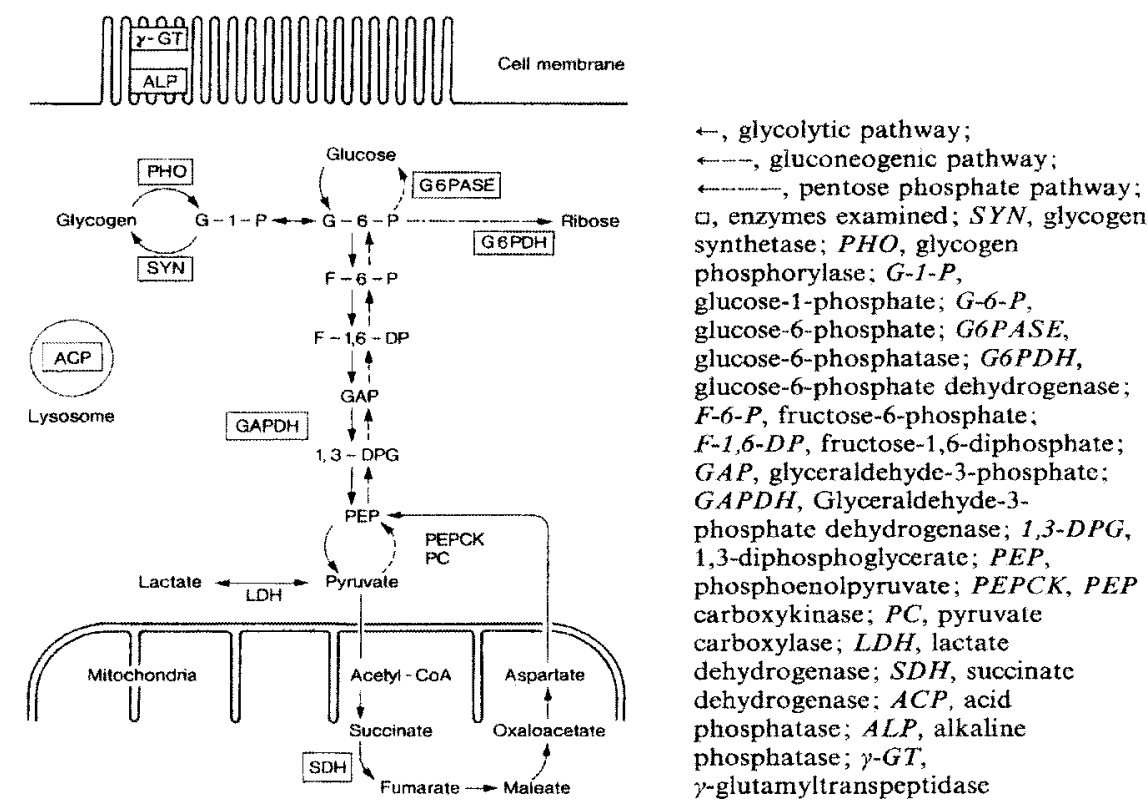

Fig. 3. Distribution of enzymes in the cytoplasm of the renal tubule (Tsuda et al., 1986) 
A

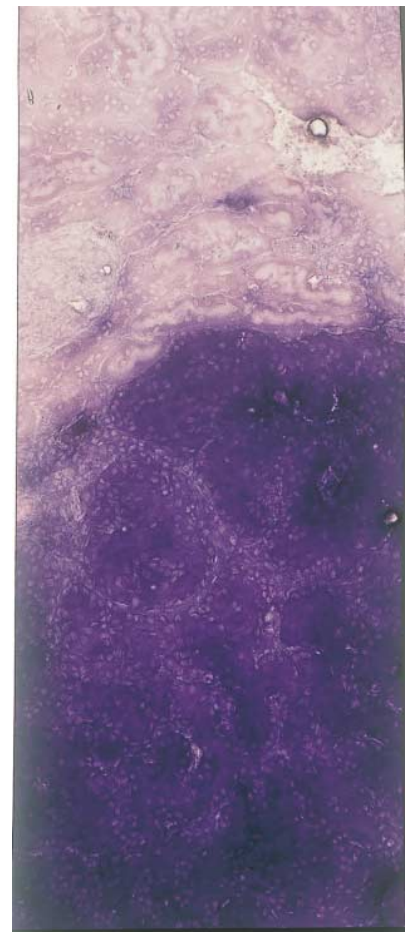

GGT

B

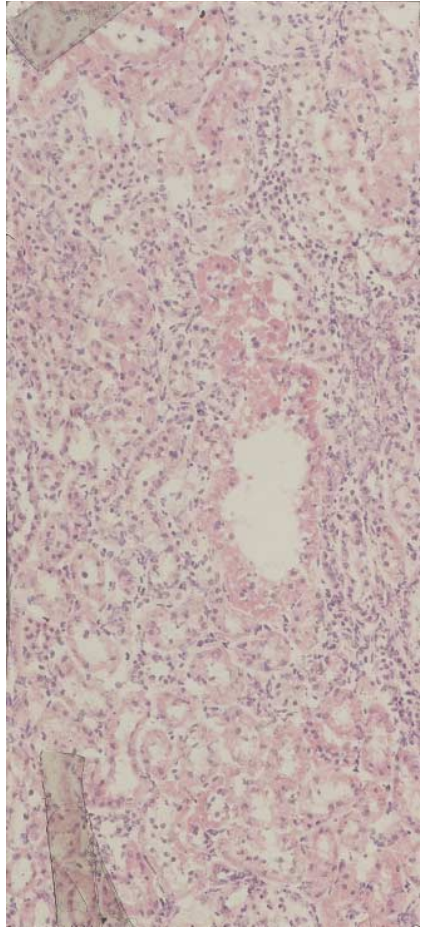

HE

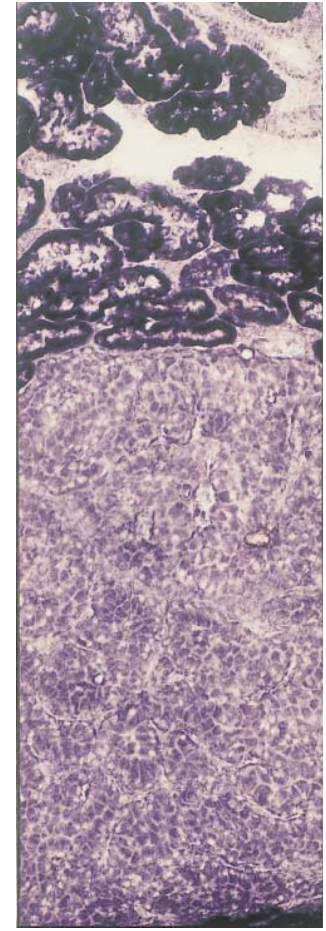

SDH

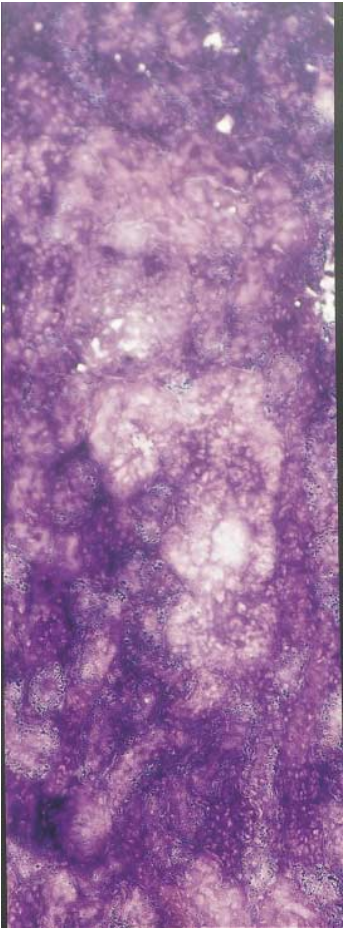

G6PD

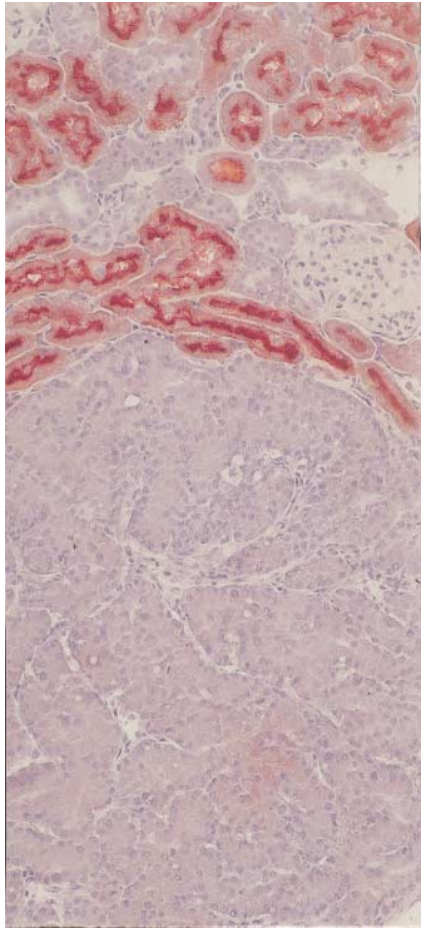

G6PD

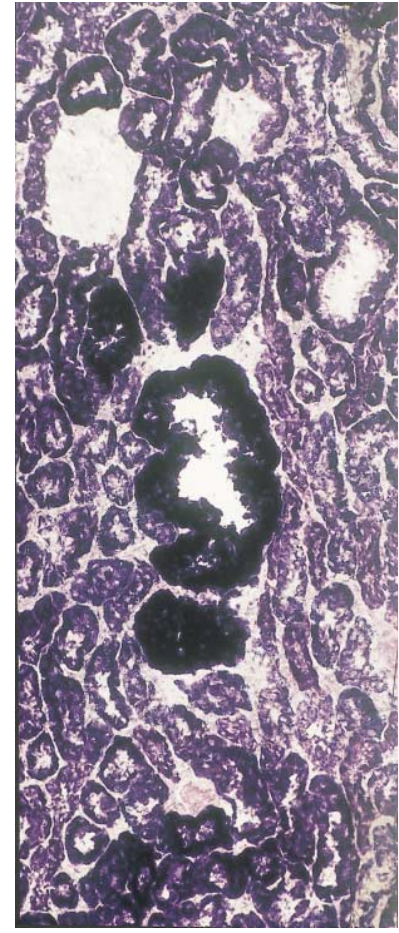

SDH

Plate 3A, B. Histochemical characteristics in renal epithelial proliferative lesions. A; histochemical characteristics of adenoma consisting of basophilic cell types stained with GGT (left), SDH (central) and G6PD (right). B; histochemical characteristics of atypical hyperplasia consisting of oncocytic cell types stained with HE (left), G6PD (central) and SDH (right). 


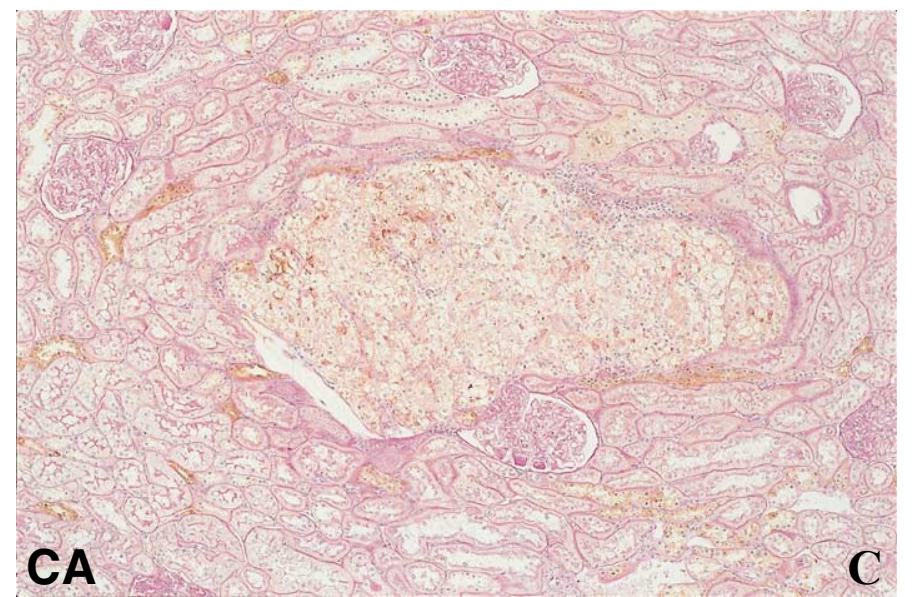

Plate 3C. Adenoma composed of clear cell type. Note positive reaction to $\mathrm{CA}$ immunohistochemically. $\mathrm{A}-\mathrm{C}$, induced cases.

Table 1. Histochemical and Immunohistochemical Pattern of Neoplastic Lesions as Compared to Normal Renal Tubules in Rat

\begin{tabular}{lcccccc}
\hline & \multicolumn{3}{c}{ Normal tubule } & \multicolumn{3}{c}{ Proliferative lesions } \\
& Proximal t. & Distal t. Collecting t. & Basophilic cell (eosinophilic) & Clear cell & Oncocytic \\
\hline SDH & + & + & \pm & $-(\downarrow)$ & $\pm(\downarrow)$ & $++(\uparrow)$ \\
G6PD & + & ++ & \pm & $++(\uparrow)$ & $\pm(\downarrow)$ & $-(\downarrow)$ \\
GGT & + a & - & - & $+\sim-(\rightarrow \sim \downarrow)$ & $-(\downarrow)$ & $-(\downarrow)$ \\
PAS & + a & - & - & $+(\rightarrow \sim \downarrow)$ & $-(\downarrow)$ & $-(\downarrow)$ \\
CA & \pm & \pm & $+\sim++$ & $-\sim \pm(\downarrow)$ & $-\sim \pm(\uparrow)$ & $+(\uparrow)$ \\
CytOx & - & + & $+\sim++$ & $-(\downarrow)$ & $+(\uparrow)$ & $+(\uparrow)$ \\
\hline
\end{tabular}

Abbreviations: -; negative, \pm ; weakly positive, + ; moderately positive, ++ ; strongly positive, a; brush border,

$\uparrow / \rightarrow / \downarrow$; increase/ no change/ decrease as compared to surrounding normal tubules

SDH; succinate dehydrogenase, G6PD; glucose-6-phosphate dehydrogenase, GGT; $\gamma$-glutamyl transpeptidase,

$\mathrm{CA}$; carbonic anhydrase type II, CytOx; cytochrome c oxidase

eosinophilic proliferative lesions with a corresponding reduction in mitochondrial respiration. On the contrary, in most oncocytic epithelial proliferative lesions, the activity of G6PD was declined, but the lesion was intensely positive for $\mathrm{SDH}$, indicating an increase of activity of the mitochondrial respiration and a marked reduction in the activity of the key enzyme of the pentose phosphate pathway.

On the double staining methods using PAS and CA or $\mathrm{CytOx}$ and stereological reconstruction of these lesions, basophilic/eosinophilic cell type lesion showed positive to PAS and transition to the proximal tubules. In the previous report by Tsuda et al., clear cell type was mostly positive to $\mathrm{CA}$ and CytOx and showed transition to both the distal and the collecting ducts. However, only one case showed transition to the proximal tubule, indicating that the origins of renal cell neoplasia for their clear cell counterpart are either proximal or distal tubules ${ }^{8,9}$. All oncocytic type was positive for $\mathrm{CA}$ and $\mathrm{CytOx}$ showing transition to the collecting tubules. Based on these histochemical and immunohistochemical features, the Working Group concluded that since epithelial proliferative lesions, such as basophilic/eosinophilic cell, oncocytic or clear cell type, are different in their cytochemical pattern and histogenesis, the cell origin of renal proliferative lesions can be clarified by the conduct of these histochemical techniques.

\section{Criteria of classification of epithelial proliferative lesions in the kidney (Plate 4)}

Based on the discussion on the histological specimen and literatures collected in the working group, the following classification was proposed by the Working Group:

\section{1) Hyperplasia, renal tubule, atypia}

This lesion can be also called as altered focus, atypical tubule, or atypical hyperplasia. As diagnostic features, it is characterized by focal proliferation of epithelial type-tubules (basophilic/eosinophilic, clear cell or oncocytic) with atypia. This lesion develops in the renal tubules with papillary, solid or cystic formation but does not have any compression of the surrounding tissues. Another type of atypical hyperplasias is called as focal proliferation of tubular type-tubules. It is a focal proliferation of epithelial or oval like cells consisting of clusters of small tubules or tubule-like structures in the interstitial tissue, without any proliferation within the tubules. 

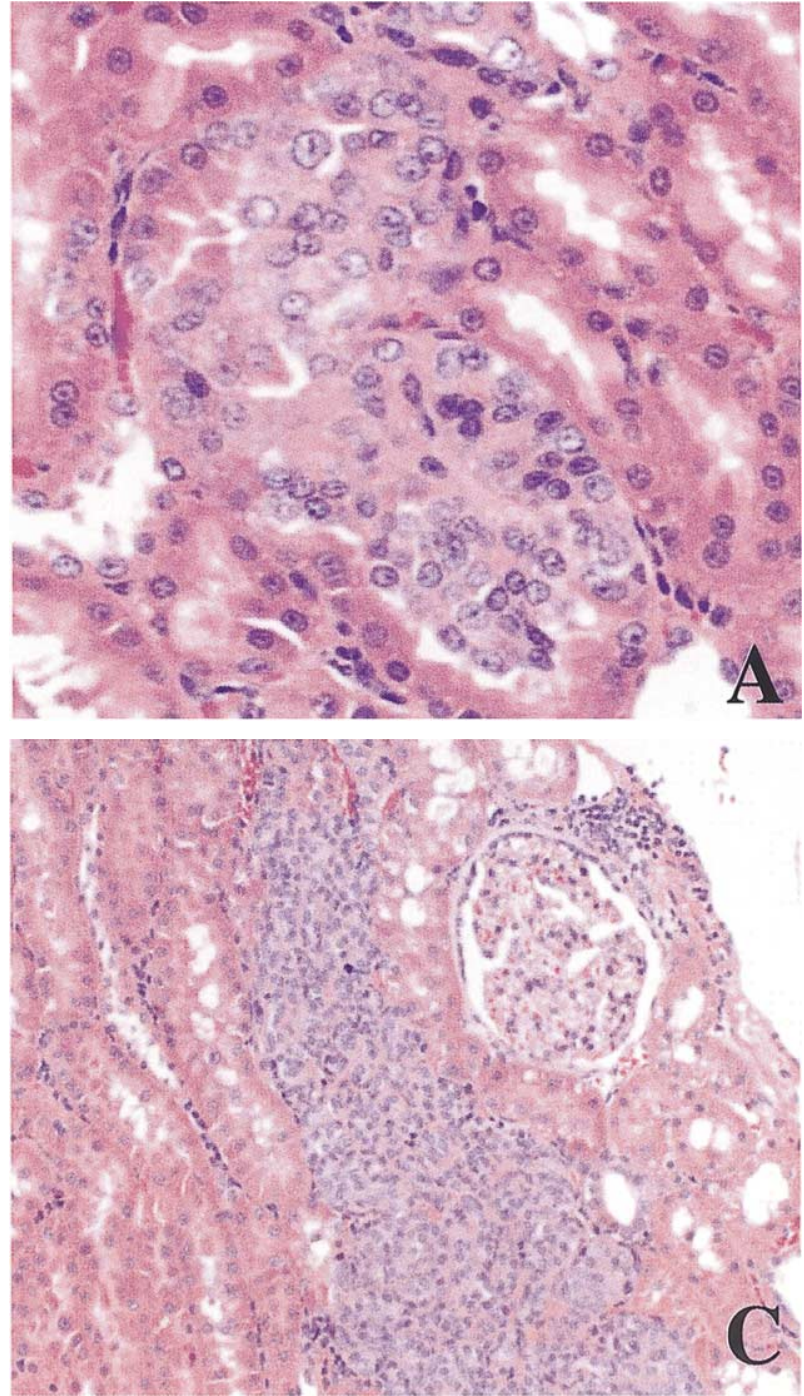

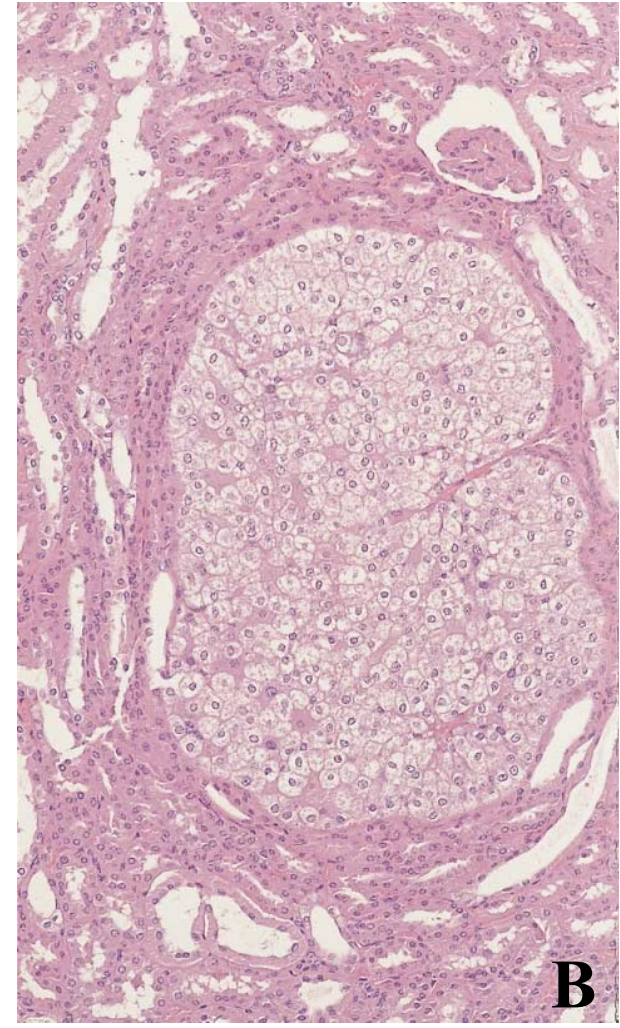

Plate 4. A; hyperplasia of renal tubule, atypia, basophilic cell type. Multilayered basophilic cells with atypia were proliferating in a renal tubule, remaining part of the tubule still showing normal structure. B; adenoma, renal tubule, clear cell type. Note the compression to adjacent renal tissue. C; adenoma composed of tubular type cells. Note the lesion was similar to adenoma of epithelial type, basophilic cell type. A-C, induced cases.
As the differential diagnosis, any compression of the surrounding tissue can not be detected in hyperplasias. As non-neoplastic lesions, simple hyperplasia is usually observed in regenerating kidneys. This simple hyperplasia is not accompanied by cytological or histological atypia in its cytoplasm.

As pointed out by the Working Group, all members felt that simple hyperplasia is a result of a reactive or regenerative change of damaged renal tubules ${ }^{10}$. Prolonged damage in the tubules might result in simple hyperplasia named as "regenerative renal tubule", which is distinguishable from atypical hyperplasia, since simple hyperplasia is usually accompanied by thickening of the basement membrane surrounding the affected tubules. This simple hyperplasia was frequently observed in the severe damaged kidney such as chronic progressive nephropathy (CPN) or chemical-induced cases (see below). On the other hand, atypical hyperplasia is often found as earlier changes of renal tumor development. Therefore, the Working Group emphasized that increased number of these atypical hyperplasias should not be missed in routine examinations.
In addition, it was recommended that chemically-induced atypical renal hyperplasia should be sub-classified into three cell types depending on their cytological cell type described above, since the sub-classification might be useful to check and identify the occurrence of compound-related changes.

\section{2) Adenoma, renal tubule}

Adenoma is characterized by focal proliferation of atypical epithelial type-tubular epithelium (basophilic/ eosinophilic, clear cell or oncocytic) with compression of the adjacent parenchyma. As the differential diagnosis between atypical hyperplasia and adenomas, multi-layered cell linings or two or more directed papillary growth with vascular-connective tissues are detected in adenomas of papillary, cystic or mixed type, but not in atypical hyperplasias. The Working Group had a opinion that it is difficult to make a diagnosis of adenomas based on the size of proliferative lesions, but proposed that in adenomas of solid type, the size exceeds more than three normal renal tubules. In addition, the Working Group emphasized that structural atypia is the good criteria for the diagnosis of 
adenomas.

As the comments pointed out by the Working Group, the members felt that it was not necessary to distinguish adenomas of the tubular type from the epithelial type, since morphological features of the adenomas advanced from hyperplasia of the tubular type was very similar to that of epithelial type and not distinguished between them. In addition, the Working Group recommends that chemicalinduced adenomas should be sub-classified into the three cell types depending on their cytological cell type described above, since the sub-classification might be useful to check and identify of the compound-related tumors.

\section{3) Adenocarcinoma, renal tubules}

Adenocarcinoma, renal tubule should be diagnosed compositely by combination of the following features: 1) Metastasis or invasive growth to the lungs or other organs is observed; 2) Mitotic figures are more frequent (for example, ten or more mitotic figures are detected at 200 times magnification); 3) Areas of hemorrhage or necrosis might be present; 4) Presence of pleomorphic or anaplastic cells.

As the comments of the Working Group, it was emphasized that tumor cells showing cellular atypia are not always increased their proliferating activity in immunohistochemical examinations using anti-proliferating cell nuclear antigen. Therefore we have to take into account that pleomorphism might not be essential feature of malignancy (Plate 5).

\section{Nephroblastoma and Mesenchymal tumor}

Since there were confusions between nephroblastomas and renal mesenchymal tumors in an exact differential diagnosis, the Working Group proposed the following
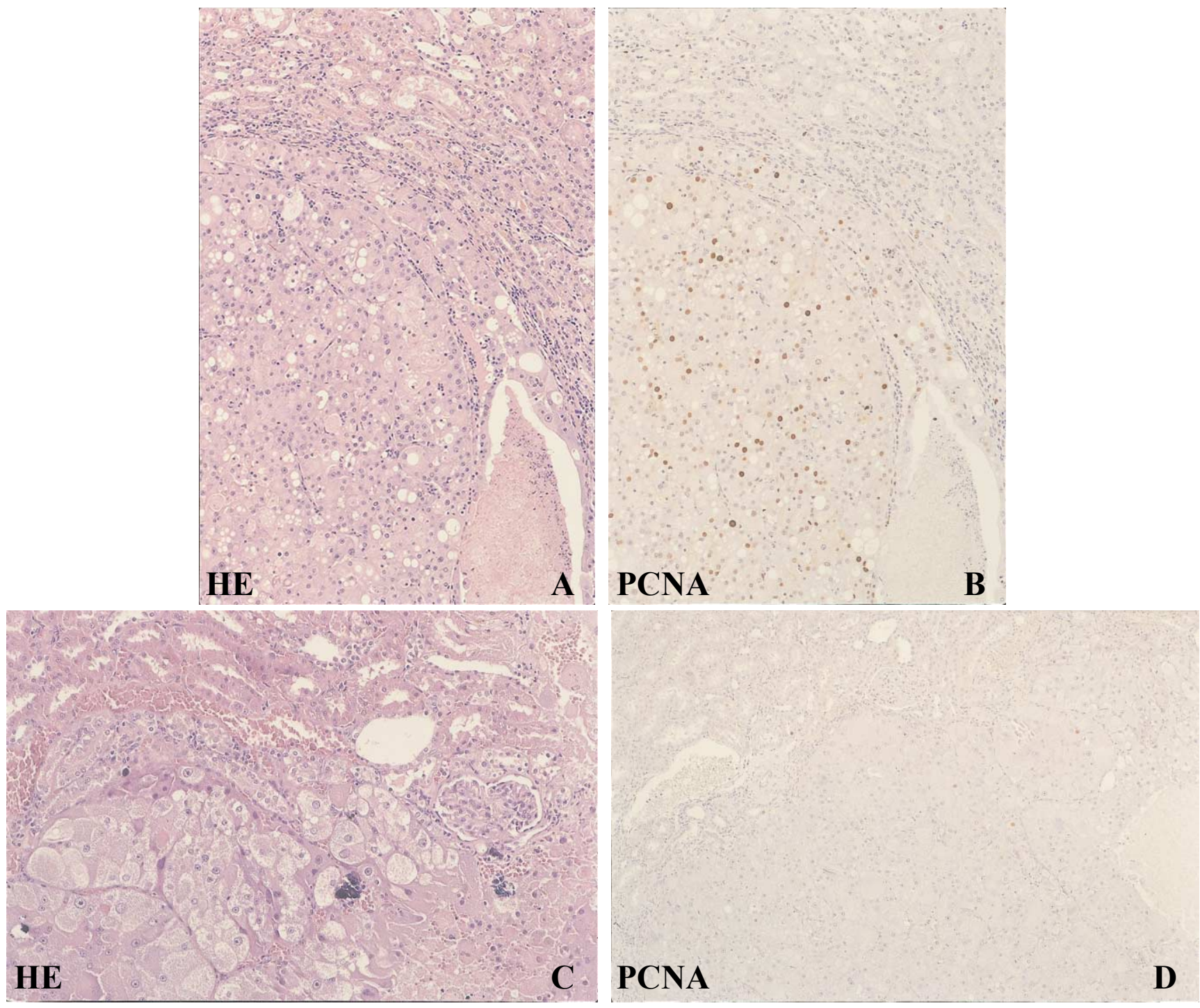

Plate 5. A; adenocarcinoma, renal tubules (HE). B; same area of the tumor stained with proliferating cell nuclear antigen (PCNA). Note a number of positive reaction of PCNA. C; adenocarcinoma with cellular atypia. D; same area of the tumor. Positive reaction to PCNA were scarcely observed. A-D, induced cases. 
criteria of classification of these tumors:

\section{1) Nephroblastoma (Plate 6)}

As the most important diagnostic features, the Working Group had a unanimous opinion that blastemal cell is essential component, and fetal glomerulus, fetal renal tubule, or organoid differentiation into other epithelial cells can be observed.

As the comments from the Working Group, the members pointed out that ciliated columnar epithelia similar to the duct of epididymis or the oviduct, both originating from the mesonephric tubules, frequently appears as the associated tissue other than the fetal glomerulus or renal tubules in spontaneous cases of non-treated rats. In addition, the members added that classification into the blastemal mesenchymal and epithelial types should be made depending on each dominant cell component.

\section{2) Renal mesenchymal tumor (Plate 7)}

As the most important diagnostic criteria, the Working Group had the same opinion that instead of blastemal cells, more than two components of mesenchyma-derived cells including a collagenous fiber, bone tissue, muscle fiber and/ or fatty tissue should be observed in renal mesenchymal tumors.

\section{Problems on the diagnosis of renal proliferative lesions in rats.}

As described below, the Working Group picked up several diagnostic problems on renal proliferative lesions that were proposed in the International Harmonization of Rat Nomenclature of the Kidney.

\section{1) Bowman's capsular hyperplasia (Plates $8 A$ and $8 B$ )}

Bowman's capsular hyperplasia should be carefully diagnosed by distinguishing from cuboidal epithelial cells of the Bowman's capsule, a kind of herniation, which is known as a physiological feature in male rats. In cases of observing hyperplasia of the adjacent proximal tubules, we should not record them as Bowman's capsular hyperplasia, but should be recorded as hyperplasia, renal tubule.

\section{2) Classification of renal tubule hyperplasia (Plates $8 C, 8 D$ and 9):}

According to the recommendation of International Harmonization of Rat Nomenclature, renal tubule hyperplasia was classified into simple (non-neoplastic) and atypical types (pre-neoplastic). Generally, simple hyperplasia of renal tubules is often seen as a reactive lesion associated with ruined renal failures or an associated lesion of an aged-related chronic progressive nephropathy (Plates $8 \mathrm{C}$ and $8 \mathrm{D})^{10}$. The Working Group proposes that the simple type of renal tubular hyperplasia in CPN is the renal tubular reactive changes including regeneration and should be included in the lesion of CPN, we recommend that only the atypical type of renal tubular hyperplasia (pre-neoplastic) should be recorded as "Hyperplasia, renal tubule, atypia" (Plate 9) in CPN. Proliferative lesions of the kidney, especially atypical hyperplasia, renal tubule, tend to increase in their occurrence with aging. However, concurrent occurrence of renal tumors and CPN in spontaneous cases is not common, suggesting that all atypical tubular hyperplasias would not progress to the tumor development, as demonstrated in the case of spontaneous liver proliferative lesions in rats.

\section{3) Intralobar nephroblastematosis as the precursor of the nephroblastoma (Plate 10)}

Persistence of the embryonal tissue in the kidney of a human is known as nodular renal blastema or the nephroblastematosis. These lesions are considered to be related to the tumorigenesis of nephroblastomas in human. The similar lesion was recently reported in the rats as "intralobar nephroblastematosis"11. The recognition that an intralobar nephroblastematosis is the precursor of the nephroblastomas is necessary.

\section{Conclusion}

The Working Group of JSTP for the proliferative lesions of the kidney in rats and mice recognized that both of spontaneous and chemical-induced lesions could be categorized by the use of the same diagnostic criteria and classification based on cytological characteristics as described above. The results of histochemical and immunohistochemical examinations indicate that the origins of epithelial proliferative lesions are proximal tubules for basophilic cell type, either proximal or distal renal parts for their clear counterpart, and collecting ducts for oncocytic ones, whereas the origin of clear cell type is still controversial. Based on the discussion, we recommend that modified classification of renal proliferative lesions (Table 2 ), and our comments to the final recommendation of International Harmonization of Rat Nomenclature of the Kidney proposed by the group of STP, WHO/IARC/RITA and NACAD in 2000 were shown in Table 3. Among these comments, what we intend to point out is that simple

Table 2. Classification of Renal Proliferative Lesions (JSTP)

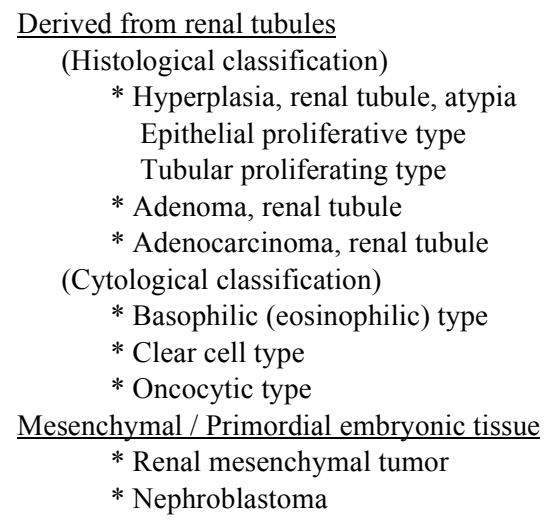



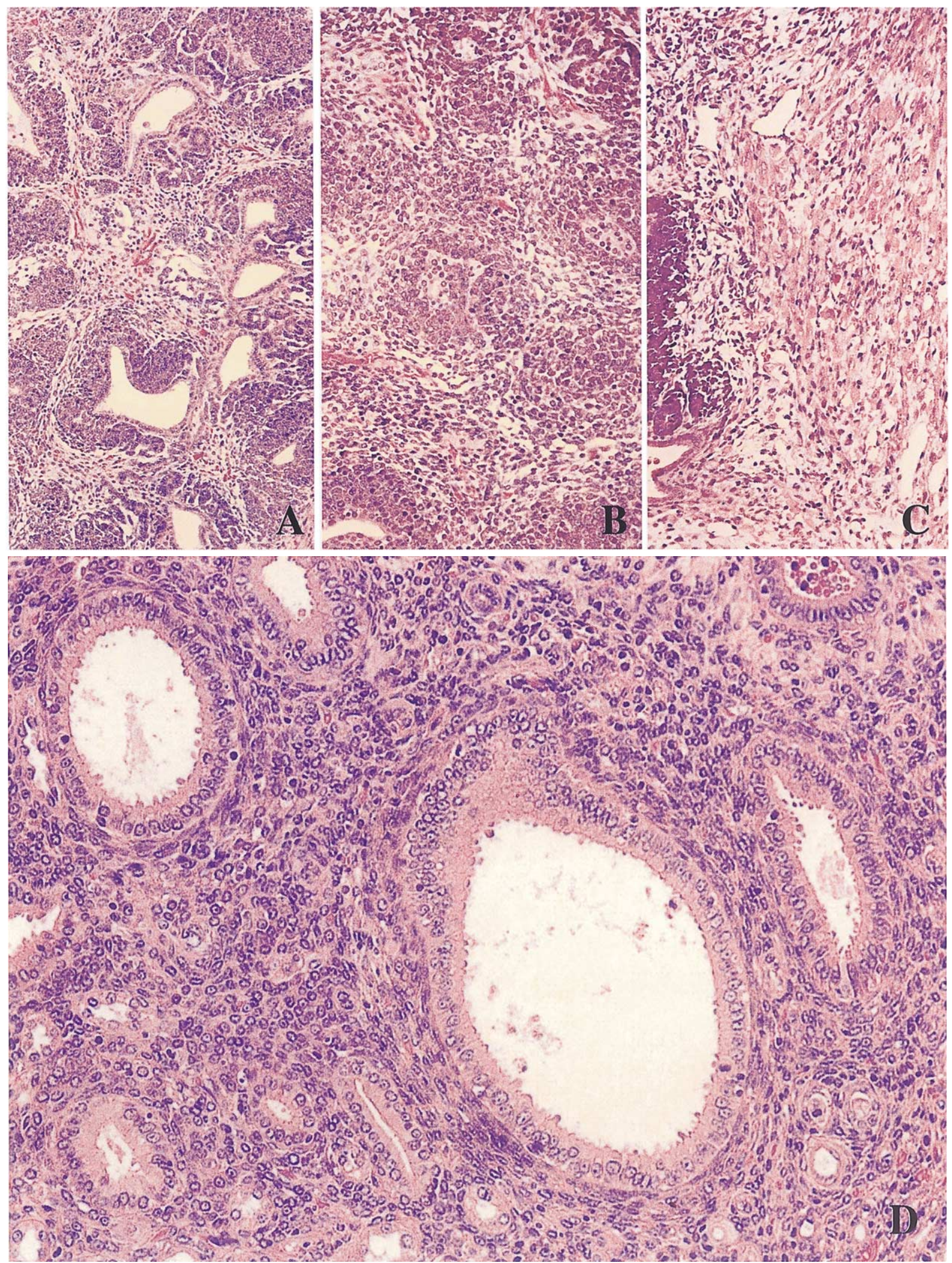

Plate 6. Nephroblastoma. It consists of epithelial components including fetal glomerulus and renal tubule (A); blastemal cells (B) and mesenchymal tissue (C). D; nephroblastoma. Ciliated columnar epithelium extremely similar with ducts of the epididymis or oviducts often appeared. A-D, Spontaneous cases. HE stain. 

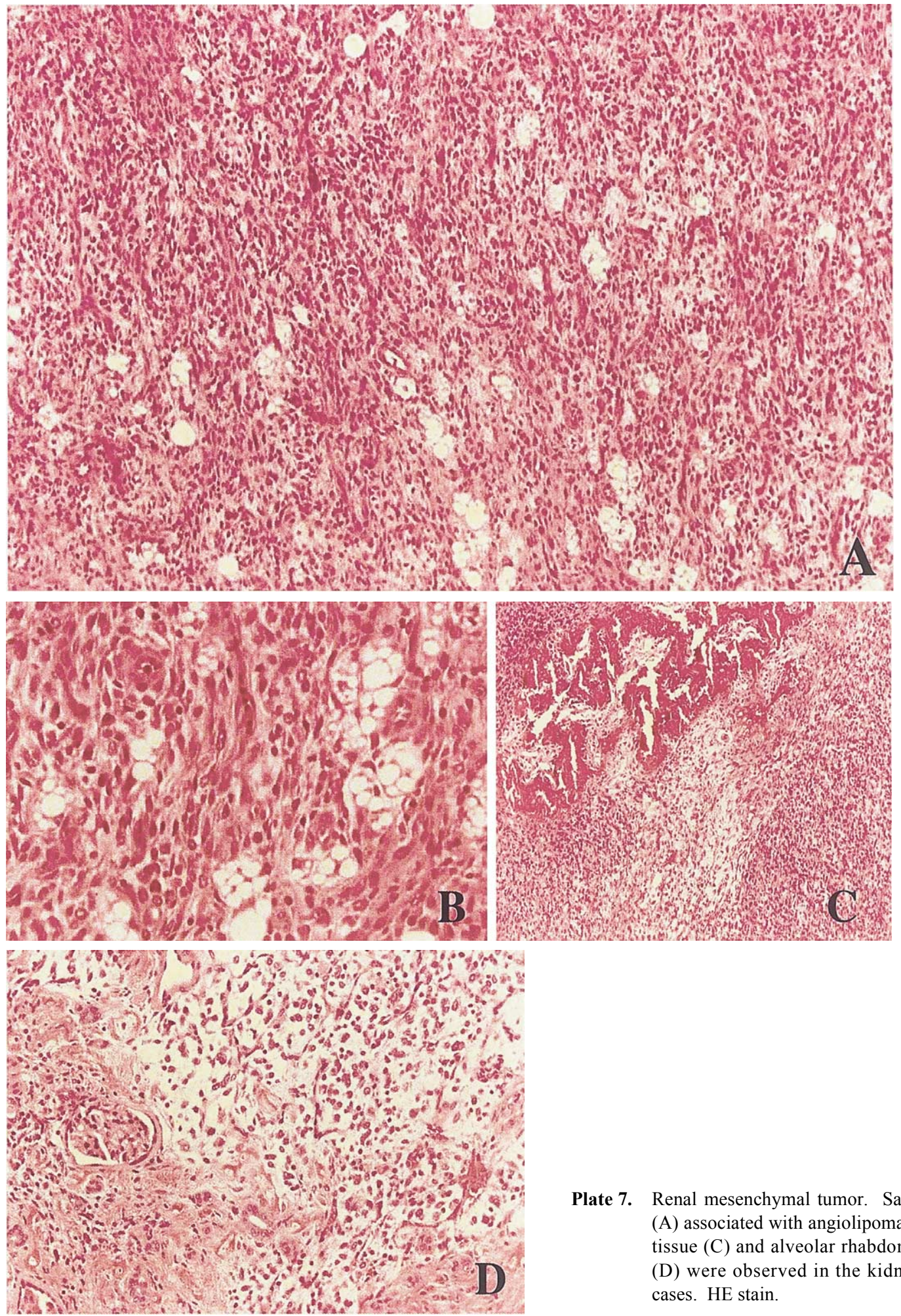

Plate 7. Renal mesenchymal tumor. Sarcomatous spindle cells (A) associated with angiolipomatous tissue (B), osseous tissue $(\mathrm{C})$ and alveolar rhabdomyosarcoma-like tissue (D) were observed in the kidney. A-D, spontaneous cases. HE stain.

hyperplasia observed in chronic progressive nephropathy (CPN) should be considered as a sequential alterations of $\mathrm{CPN}$, and should not be diagnosed as independent findings of CPN. Therefore, it is recommended that only the atypical type of renal tubular hyperplasia (pre-neoplastic) should be recorded as "hyperplasia, renal tubule, atypia". In addition, we presented a new category of renal tubular hyperplasia that is classified into epithelial or tubular types, and it was emphasized that especially the latter should not be overlooked in routine examinations for carcinogenicity studies. As described above, there are still several points that need to be clarified in the internationally harmonized 


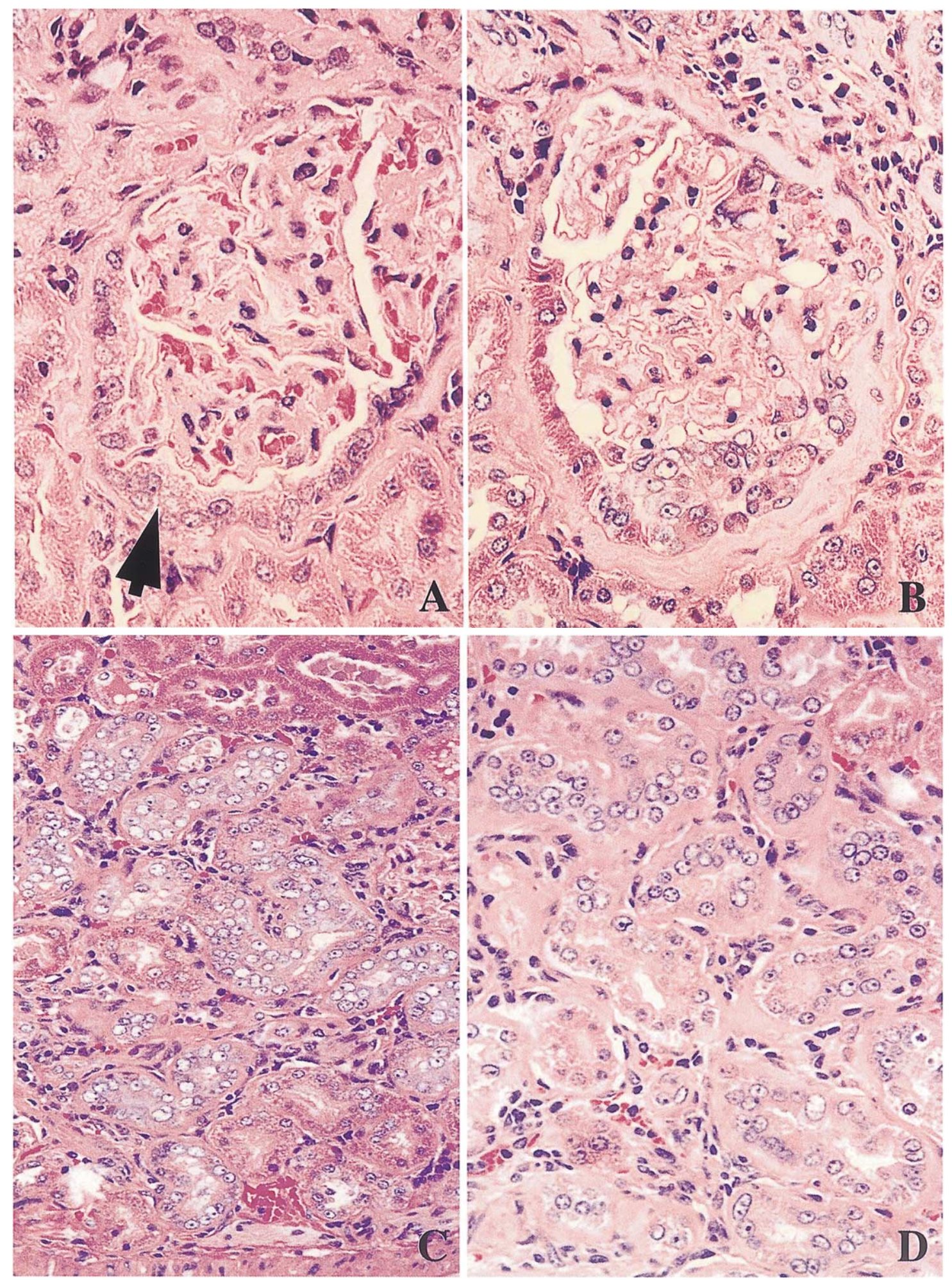

Plate 8. A, B, bowman's capsular hyperplasia. Parietal lining cuboidal cells were increased (arrow). C, D, simple hyperplasia of renal tubule. Number and cell density of renal tubules were increased within single cell layer. These lesions associated with aging chronic progressive nephropathy. A-D, spontaneous cases. HE stain. 

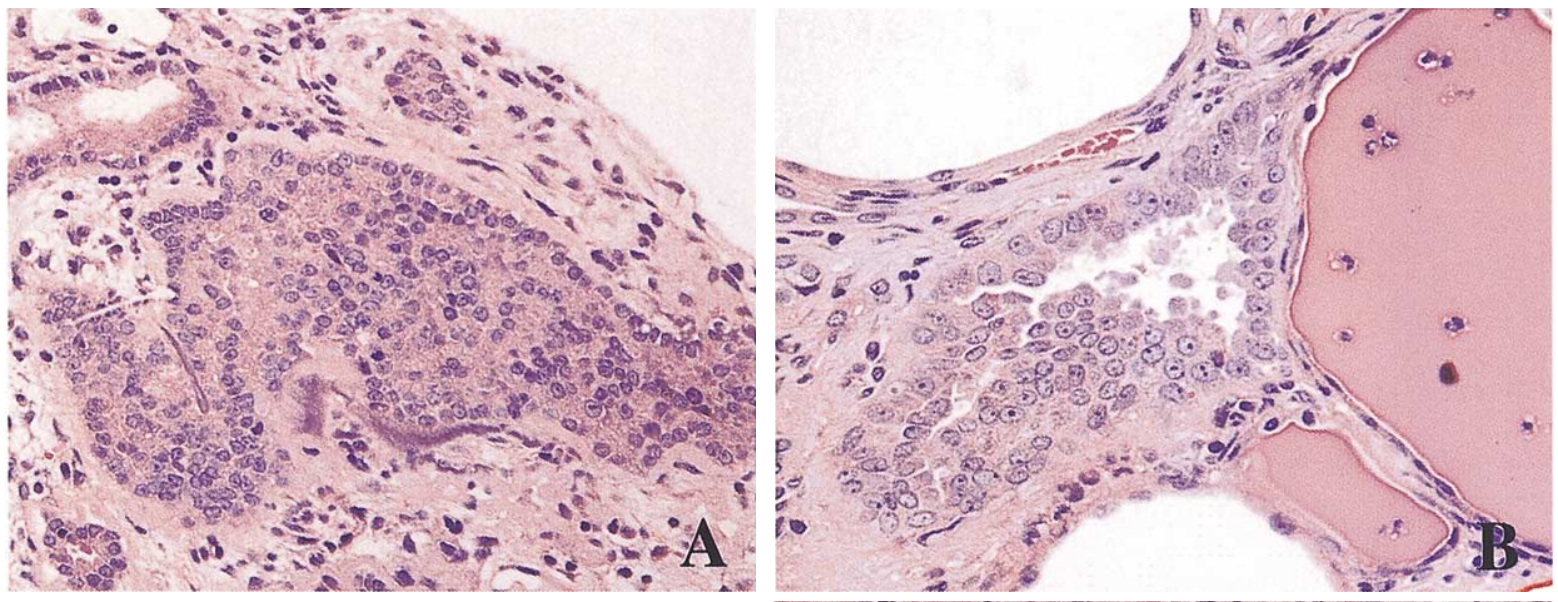

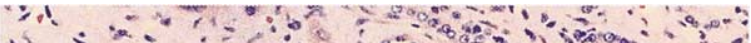

40 and
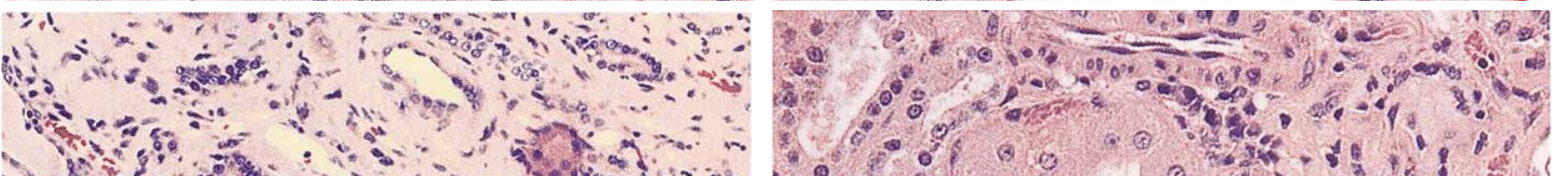

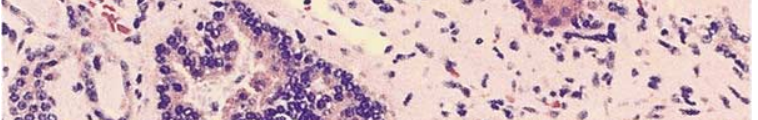

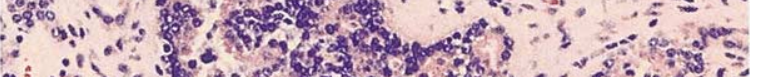

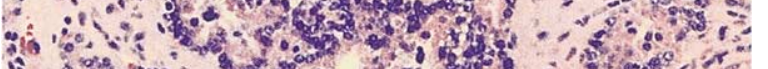
3.

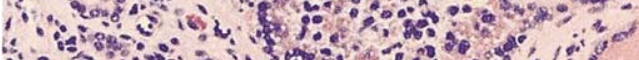

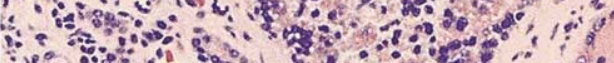
1. - 0 -
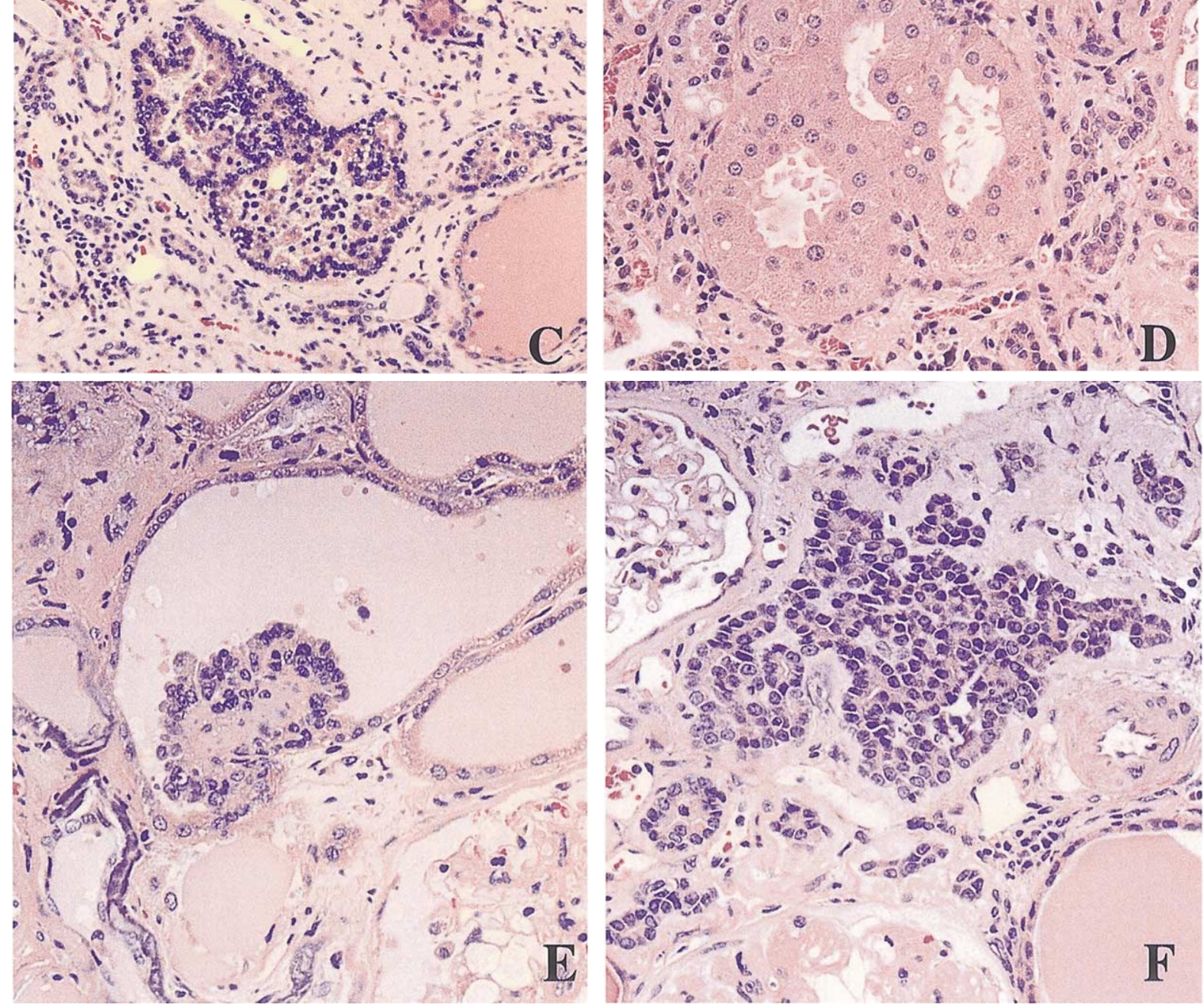

Plate 9. Hyperplasia, renal tubule, atypia. Cellular atypia was found, and number and cell density of renal tubule were increased with multilayered structure. These lesions also associated with aging chronic progressive nephropathy. These lesions were classified into epithelial type (A-E) and tubular type (F). Note that F is similar to Plate 1D. A-F, spontaneous cases. HE stain. 


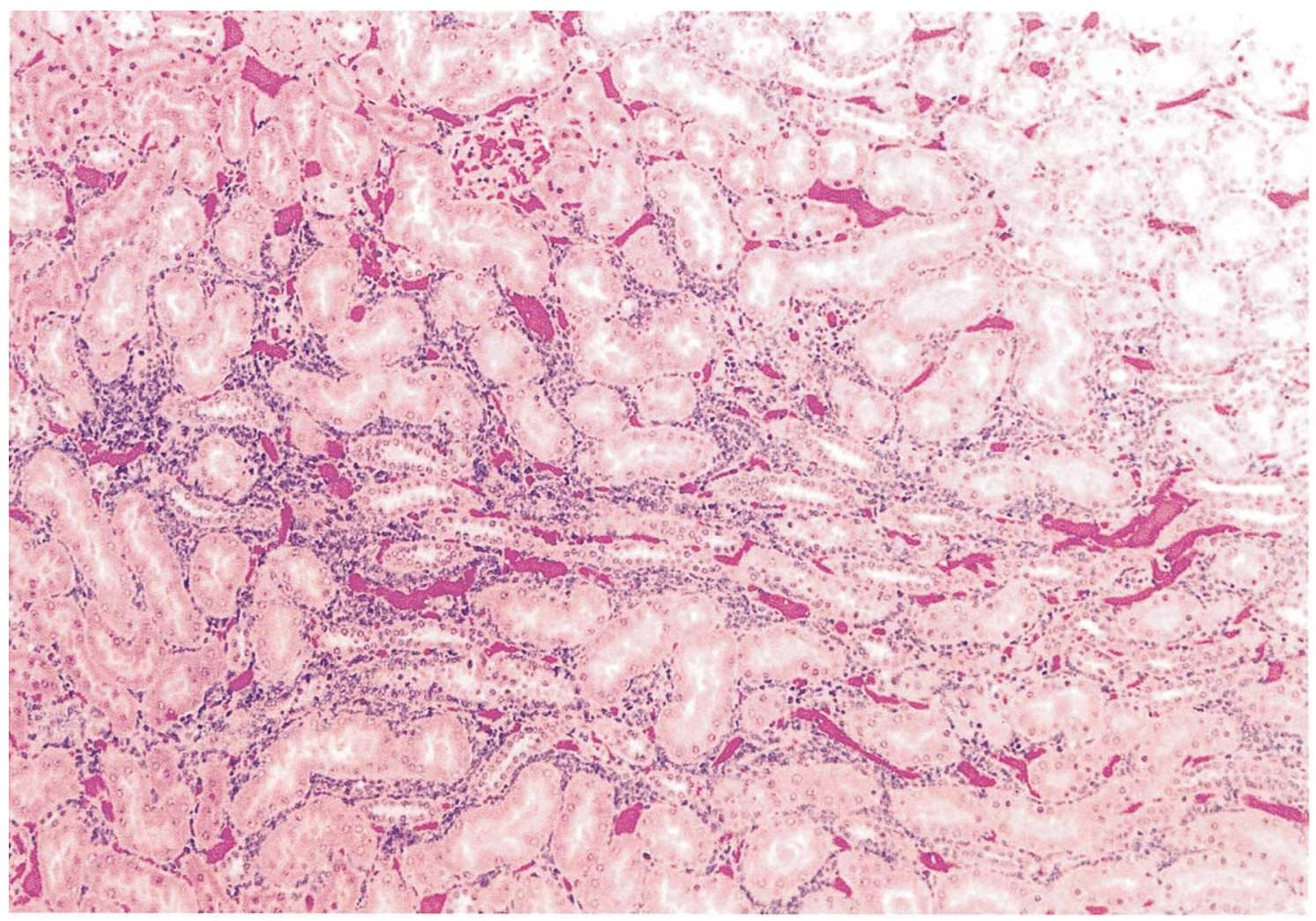

Plate 10. Intralobar nephroblastematosis. Blastemal cells with rich in chromatin were spread in intralobar interstitium.

Table 3. Proposal Classification of the Working Group to International Harmonization of Rat Nomenclature

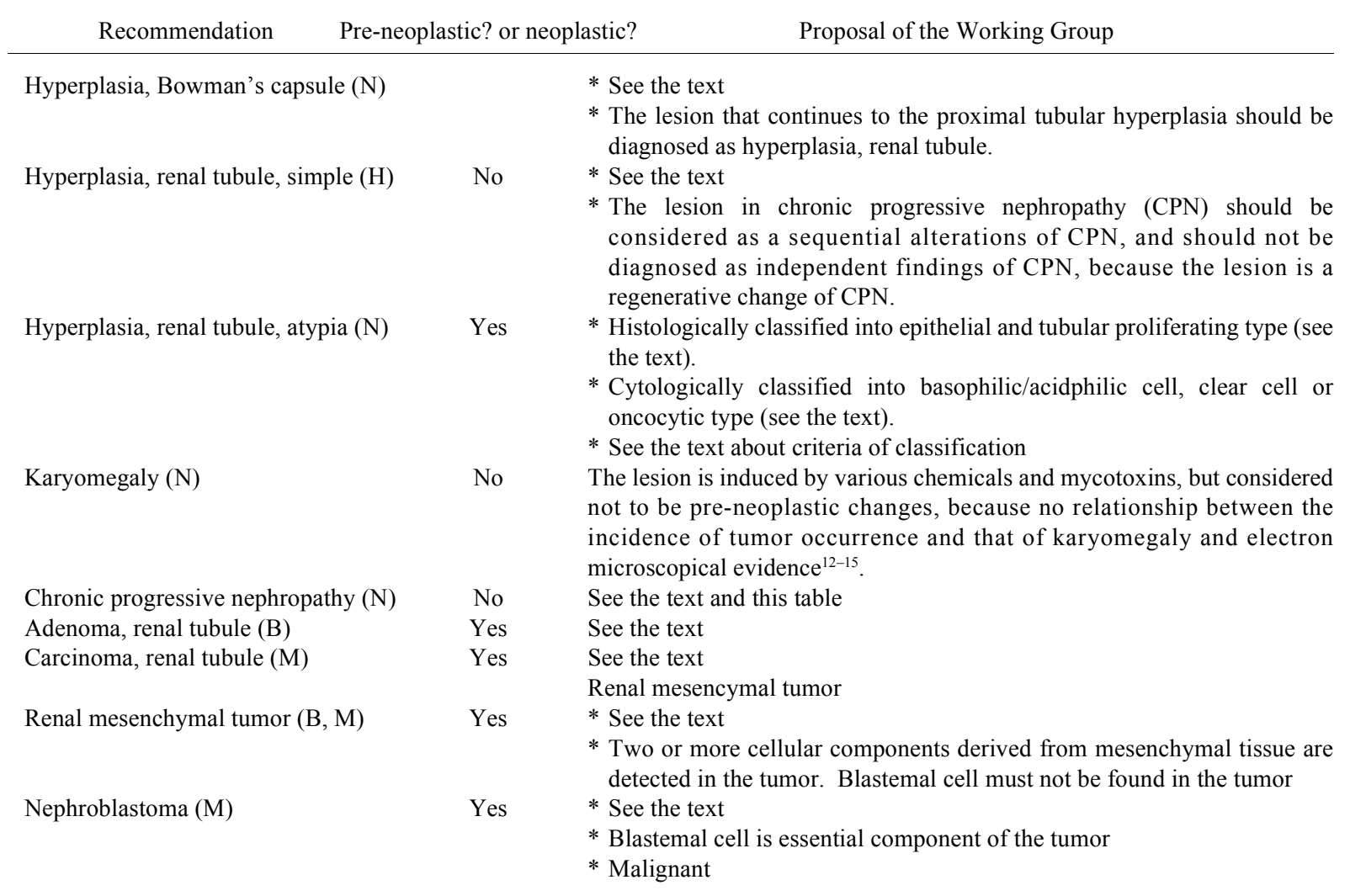

(N); non-neoplastic, (H); hyperplasia (B); benign, (M); malignant. 
nomenclature recommended. Further collaborative studies taking into account our comments and modified classification are necessary for the better internationally harmonized nomenclature of renal proliferative lesions in rodents.

Acknowledgements: The authors greatly appreciate the exellent advice of Dr. Akihiko Maekawa, Sasaki Institute to this manuscript. The authors also wish to thank Dr. Tomoyuki Shirai, Head of the Educational Committee of the Japanese Society of Toxicologic Pathology (JSTP) and Dr. Kunio Doi, Editor of JSTP for giving us the opportunity to publish our outcome that was presented in the second meeting for the Continuing Education Seminar for Toxicologic Pathologists on November 29, 2001.

\section{Reference}

1. Hard GC. Renal carcinogenesis. Rat. In: Urinary system. TC Jones, U Mohr, RD Hunt (eds), Berlin: Springer-Verlag, 4549, 1986

2. Kurata Y, Diwan BA, Uno H, Rice JM, and Ward JM. Pathology of preneoplasitc and neoplastic renal tubular lesions induced in F-344 rats by sodium barbital, a nongenotoxic renal carcinogen and nephrotoxin. Toxicol Pathol 1993; 21: 35-45.

3. Hard GC, Howard PC, Kovatch RM, and Bucci TJ. Rat kidney pathology induced by chronic exposure to fumonisin B1 includes rare variants of renal tubular tumor. Toxicol Pathol 2001; 29: 379-386.

4. Hard GC, Boorman GA, and Wolf DC. Re-evaluation of the 2-year chloroform drinking water carcinogenicity bioassay in Osborne-Mendel rats supports chronic renal tubule injury as the mode of action underlying the renal tumor response. Toxicol Pathol 2000; 53: 237-244.

5. Tsuda H, Matsumoto K, Iwase T, Nishida Y, and Baba H. Enhanced neoplastic lesion development with adenineinduced experimental multicystic nephropathy by adenine a model system for the analysis of renal tumor generation in long-term hemodialysis patients. Cancer Lett 1994; 83: 105110.

6. Hard GC. Tumours of the kidney, renal pelvis and ureter. In: Pathology of Tumours in Laboratory Animals. Volume 1 -
Tumours of the Rat. U Turusov and U Mohr (eds), Lyon: International Agency for Research on Cancer, 301-344, 1990.

7. International Agency for Research on Cancer, World Health Organization. International Classification of Rodent Tumours, Part I - The Rat, 3. Urinary System. U Mohr (Editor-in-Chief), Lyon: International Agency for Research on Cancer, 1-46, 1992.

8. Tsuda H, Hacker HJ, Katayama H, Masui T, Ito N, and Bannasch P. Correlative histochemical studies on preneoplastic and neoplastic lesions in the kidney of rats treated with nitrosamines. Virchows Arch [Cell Pathol] 1986; 51: 385-404.

9. Tsuda H, Iwase T, Matsumoto K, Ito M, Hirono I, Nishida Y, Takasuka N, Iwahori Y, Ota T, Kim DJ, and Kadenbach B. Histogenetic stereological reconstruction of rat basophilic, clear, and oncocytic neoplastic renal cell lesions using carbonic anlydrase type II - PAS double-stained sections. Toxicol Pathol 1998; 26: 769-776.

10. Ozaki K, Mahler JF, Haseman JK, Moomaw CR, Nicolette ML, and Nyska A. Unique renal tubule changes induced in rats and mice by the peroxisome proliferator 2,4dichlorophenoxyacetic acid (2,4-D) and WY-14643. Toxicol Pathol 2001; 29: 440-450.

11. Mesfin GM. Intralobar nephroblastematosis: precursor lesion of nephroblastoma in the Sprague-Dawley rat. Vet Pathol 1999; 36: 379-390.

12. Watanabe $M$ and Nishikawa A. Kidney. In: Dokuseibyourisoshikigaku, Nagoya: the Japanese Society of Toxicologic Pathology, 247-266, 2000 (in Japanese).

13. Morgan DL, Mahler JF, Wilson RE, Moorman MP, Price $\mathrm{HC} \mathrm{Jr}$, and O'Connor RW. Toxicity of divinylbenzene-55 for B6C3F1 mice in a two-week inhalation study. Fundam Appl Toxicol 1997; 39: 89-100.

14. Yasuhara $\mathrm{K}$, Mitsumori $\mathrm{K}$, Imazawa $\mathrm{T}$, Yoshimura $\mathrm{H}$, Onodera H, Takegawa K, Takahashi M, Shirai W, and Hayashi Y. Ultrastructure and cell proliferative activities of karyomegalic alveolar epithelial cells in early pulmonary inflammatory lesions of Syrian golden hamsters induced by N-methyl-N-nitrosourethane. J Vet Med Sci 1996; 58: 825831.

15. Boorman GA, McDonald MR, Imoto $S$, and Persing $R$. Renal lesions induced by ochratoxin A exposure in the F344 rat. Toxicol Pathol 1992; 20: 236-245. 